\title{
Comparison between the performance of Algorithmic Optical Codes and Orthogonal Optical Codes in OCDMA systems
}

\author{
Jesus A. Martin-Gonzalez, Enrique Poves, Francisco J. Lopez-Hernandez \\ Dept. of Photonics Technology, Universidad Politecnica de Madrid, \\ ETSIT, Av. Complutense 28040 Madrid, Spain \\ jesus.martin@tfo.upm.es, kike@tfo.upm.es and dxtn@tfo.upm.es
}

\begin{abstract}
A novel method to be used in OCDMA systems is exhaustively described in this paper. It uses Algorithmic Optical Codes (AOCs). They are based on the signature sequence idea. However patterns are constantly changing. A common seed for pseudo-random sequence is the signature element.

In this paper a derivation of the probability of error due to the multiple-access interference is provided. An other contribution of this work is also the comparison between the AOCs and the Orthogonal Optical Codes (OOCs) with (auto- and cross-) correlation equal to one. Attending to the cardinality limitation problem observed in OOCs, the comparison is focused on the number of users allowed by the two different systems aforementioned. As result we state that a system using AOCs allows more users than one using OOCs when the codes are long enough.

Index Terms-Algorithmic Optical Codes, Optical codes, Orthogonal Optical Codes, Optical code-division multipleaccess (OCDMA), Spread Spectrum.
\end{abstract}

\section{INTRODUCTION}

Modulation in additive and incoherent Optical CodeDivision Multiple-Access (OCDMA) systems has traditionally been resolved by Orthogonal Optical Codes (OOCs) [1]. These families are described by a set of four numbers [2], [3]: $\left\{L, w, \lambda_{a}, \lambda_{c}\right\}$ where $(L)$ is the length of the code, $(w)$ is the weight, $\left(\lambda_{a}\right)$ is the autocorrelation and $\left(\lambda_{c}\right)$ is the cross-correlation. The maximum number of supported users is sometimes limited by the low cardinality of the family. This fact conjunction with the system performance are the key of the family design.

Different systems have been proposed based on several families of optical codes, e.g. optical orthogonal codes (OOCs) [1]-[4], prime sequences [5]-[7] or $2^{n}$ prime codes [8]-[10]. All of them fix a signature for each user. This code is the same during the whole transmission. Recently, [11]-[13], a new family of codes with no practical cardinality limitations have been introduced. The features of this family, Random Optical Codes (ROCs), make them adequate to be used in same specific applications. However, as Salehi highlights in
[14], the design of optical codes families still plays an important role in OCDMA systems.

In this paper we propose a novel method to spread the spectrum: Algorithmic Optical Codes (AOCs). The derivation of its probability of error and a comparison between the cardinality of AOCs and Orthogonal Optical Codes (OOCs) is the main contribution of the work.

AOCs keeps the essential idea of DirectSequence/Spread-Spectrum (DS/SS) used also in OOCs. Both families spread the spectrum in the same way. During the transmission of an 1-data bit, $w$ pulses are sent in the on-chips of the code. No pulses are sent during the transmission of a 0 -data bit. One bit is sent each $L$ chips. However in the systems using AOCs the length of any bit is typically different than other. It means that there is not a code signature, but there is an algorithmic signature.

The algorithm to build the code is described as follows. A Bernoulli random variable is built for each chip in the emitter and the receiver. It is created by the same method using a common seed in both devices. It guarantees the same sequence in a transmitter and its respective receiver. This random variable decides if the chip is or not a marked chip. Notice that these marked chips are analogous to the on-chips in OOCs. The seed of the pseudo-random sequence guarantees that emitter and receiver have the same marked chips. The bit transmission is finished when a number of marked chips equal to $w$ are happened. In general an AOCs family is a set of $\{0,1\}$ sequences which are built following the pseudo-random algorithm above described.

The rest of this paper is organized as follows. The parameters and design of an OCDMA system using AOCs are described in Sec. II. Its performance is analyzed by the derivation of the probability of error in Sec. III. Moreover, a comparison between theoretical and simulated values of the probability of error is shown in Sec. IV. In addition a comparison of the cardinality of a system using AOCs and OOCs is shown in Sec. V. Finally the conclusions are presented in Sec. VI. 


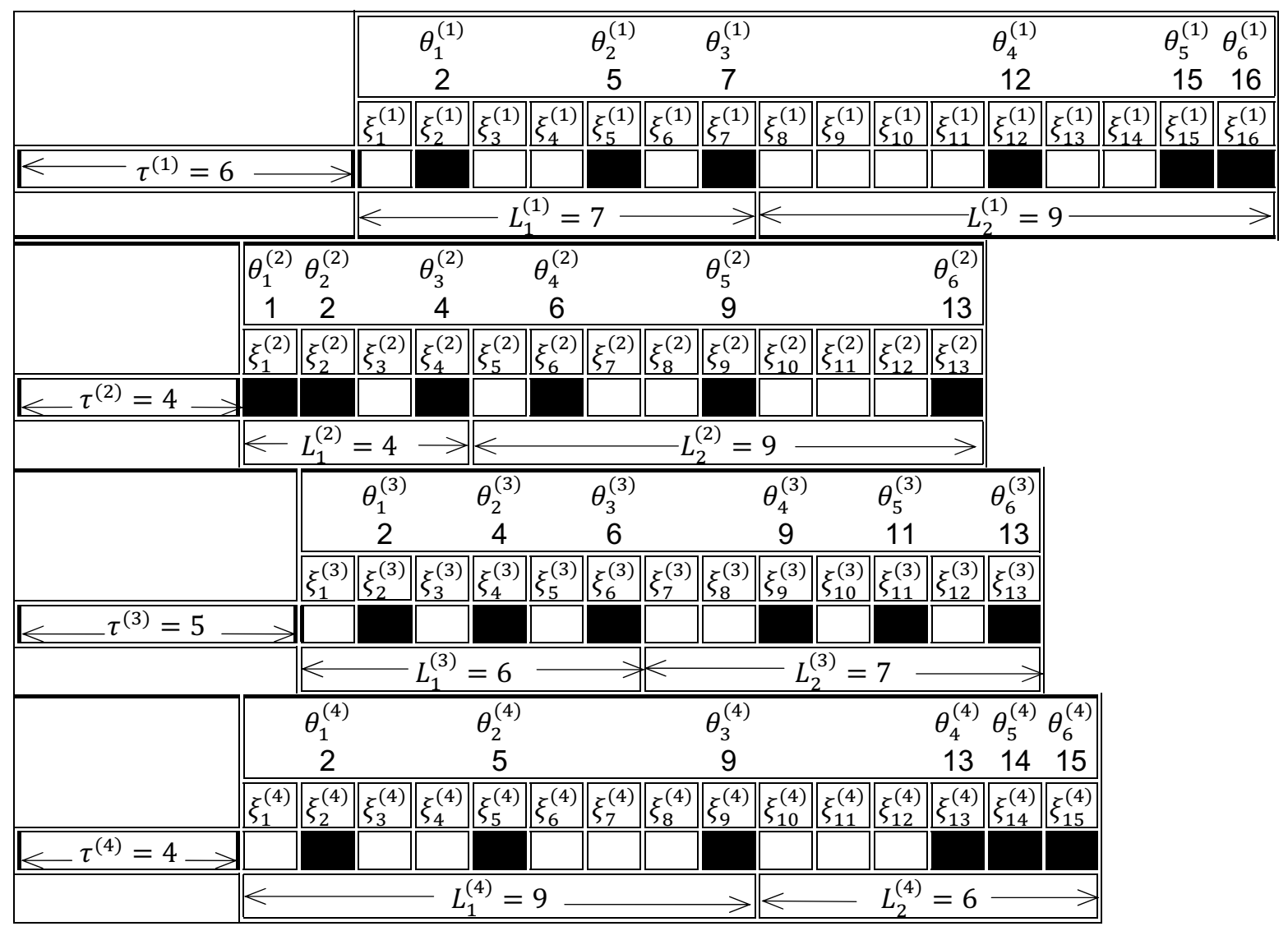

Fig. 1. Scheme of the model.

\section{SYSTEM MODEL}

Our model is an on-off keying (OOK) OCDMA system. We assume phase synchronism between the emitter and the intended receiver. However others channels could be randomly delayed. Perfect chip synchronization is assumed, i.e. frequency locking. Thus, the channels are asynchronous incoherent and additives. The number of users is $N+1$.

The codes to spread the spectrum are built as follows. We assume an infinite pseudo-random sequence of Bernoulli random variables (r.v.), i.e. $\left\{\xi_{i}^{(k)}\right\}_{i=1}^{\infty}$, for each channel. It is identically repeated by encoder and decoder starting in a common seed.

$$
\xi_{i}^{(k)}=\left\{\begin{array}{c}
\text { Yes } \\
\text { No }
\end{array} i \in \mathbb{N}, k=\{1, \ldots, N+1\}\right.
$$

Hence, $\left\{\xi_{i}^{(k)}\right\}_{i=1}^{\infty}$ are Bernoulli distributions with:

$$
P M F=\left\{\begin{array}{l}
P\left(\xi_{i}^{(k)}=\mathrm{Yes}\right)=p \\
P\left(\xi_{i}^{(k)}=\mathrm{No}\right)=1-p
\end{array}\right.
$$

where $p$ is the frequency of "Yes".

In order to track the chips where $\xi_{i}^{(k)}$ is "Yes" (marked chips), the information is stored in numerical variables $\left\{\theta_{n}^{(k)}\right\}_{n=1}^{\infty}$. Any $\theta_{n}^{(k)}$ is defined as the $n^{t h}$ marked chip for the user $(k)$, i.e.

$\theta_{n}^{(k)}=j, n=1$,

when $\xi_{1}^{(k)}=$ No $\cap \ldots \cap \xi_{j-1}^{(k)}=$ No $\cap \xi_{j}^{(k)}=$ Yes $\theta_{n}^{(k)}=j, n=\{2, \ldots, \infty\}$,

when $\xi_{\theta_{(n-1)}^{(k)}}^{(k)}=$ No $\cap \ldots \cap \xi_{j-1}^{(k)}=$ No $\cap \xi_{j}^{(k)}=$ Yes

The weight $(w)$ of the family is the number of marked chips used to transmit a bit. The length $(L)$ of the code is the number of chips that the sequence has to wait before to obtain $w$ marked chips. Furthermore, $L$ is the position of the $w^{t h}$ marked chip, i.e. $L=\theta_{w}$. Finally, let $\left\{x_{i}^{(k)}\right\}_{i=1}^{L_{i}^{(k)}}$ and $\left\{r_{i}^{(k)}\right\}_{i=1}^{L_{i}^{(k)}}$ denote the emitted and 
received signals in the $i^{t h}$ chip by the user $k$. A scheme of the system is shown in Fig. 1.

Notice that $L_{i}^{(k)}$ are random variables. Thus, the bit rate is it too. It is not a problem because we know its distribution. Therefore, we can provide confidence intervals for $L_{i}^{(k)}$, besides its expectation. The distribution of $L_{i}^{(k)}$ is equal for any user and any code. In all of them, $L_{i}^{(k)}$ measures the number of trials until the $w^{\text {th }}$ success of a Bernoulli random variable. Thus, $L_{i}^{(k)} i \in \mathbb{N}, \quad k=\{1, \ldots, N+1\}$ have Negative Binomial distribution with parameters $w$ and $p$.

\section{Performance Analysis}

Without loss of generality, we compute the probability of error for the first user. Thus, the notation can be simplified as follows:

$$
\begin{aligned}
\theta_{i} & =\theta_{i}^{(1)} \\
x_{i} & =x_{i}^{(1)} \\
r_{i} & =r_{i}^{(1)}
\end{aligned}
$$

The first step in the derivation is:

$$
P_{e}=\frac{1}{2} P(\text { error } \mid 0)+\frac{1}{2} P(\text { error } \mid 1)
$$

The second term is equal to zero [15].

$$
\begin{aligned}
P_{e} & =\frac{1}{2} P(\text { error } \mid 0) \\
& =\frac{1}{2} P(R=1 \mid 0) \\
& =\frac{1}{2} P\left(r_{\theta_{1}}=1, \ldots, r_{\theta_{w}}=1 \mid 0\right)
\end{aligned}
$$

The response in different positions are independent and identically distributed. Thus, (8) is written as follows,

$$
\begin{aligned}
P_{e} & =\frac{1}{2} P\left(r_{\theta_{1}}=1, \ldots, r_{\theta_{w}}=1 \mid 0\right) \\
& =\frac{1}{2} P\left(r_{\theta_{1}}=1 \mid 0\right) \cdot \ldots \cdot P\left(r_{\theta_{w}}=1 \mid 0\right) \\
& \left.=\frac{1}{2}\left(P\left(r_{i}=1 \mid 0\right)\right)\right)^{w} \\
& =\frac{1}{2}\left(1-P\left(r_{i}=0 \mid 0\right)\right)^{w}
\end{aligned}
$$

There is only one way to listen 0 in the observed position ( $i$ ). It happens when all the emitters have transmitted 0 in this position. It is well-known that the first user is transmitting 0 . Hence, the event only depends on the other users,

$$
P\left(r_{i}=0 \mid 0\right)=P\left(x_{i}^{(2)}=0, \ldots, x_{i}^{(N+1)}=0\right)
$$

The transmission is independent for every user. Thus, (10) can be written as,

$$
P\left(r_{i}=0 \mid 0\right)=P\left(x_{i}^{(2)}=0\right) \cdot \ldots \cdot P\left(x_{i}^{(N+1)}=0\right)
$$

The expression (11) can be written as follows because the probability of transmission of a zero in chip $i$ is the same for any emitter.

$$
\begin{aligned}
P\left(r_{i}=0 \mid 0\right) & =\left(P\left(x_{i}^{(j)}=0\right)\right)^{N} \\
& =\left(1-P\left(x_{i}^{(j)}=1\right)\right)^{N}
\end{aligned}
$$

Finally, the probability of on-chip transmission by any $j$ emitter, in any chip $i$, is the half of being a marked chip i.e. $P\left(\xi_{i}^{(j)}=\right.$ Yes $)$. We assume that only the half of the transmitted bits are 1-data bits. Then, the expression for (12) is:

$$
\begin{aligned}
P\left(r_{i}=0 \mid 0\right) & =\left(1-P\left(x_{i}^{(j)}=1\right)\right)^{N} \\
& =\left(1-P\left(\xi_{i}^{(j)}=\text { Yes }\right)\right)^{N} \\
& =\left(1-\frac{p}{2}\right)^{N}
\end{aligned}
$$

Combining (8) and (13), the expression for $P_{e}$ is as follows,

$$
P_{e}=\frac{1}{2}\left(1-\left(1-\frac{p}{2}\right)^{N}\right)^{w}
$$

The model is easily interpreted if we consider the expectation of $L$ as a parameter of the system. In Sec. II we stated that $L$ is a Negative Binomial distribution with parameters $w$ and $p$, thus $E[L]=\frac{w}{p}$. Notice that $p$ can be tailored in order to get the desirable $E[L]$ for our system, i.e. $p=\frac{w}{E[L]}$. Therefore the expression for $P_{e}$ depends on $E[L], w$ and $N$.

$$
P_{e}=\frac{1}{2}\left(1-\left(1-\frac{w}{2 E[L]}\right)^{N}\right)^{w}
$$

\section{Discussion}

In this section we present the theoretical values for $P_{e}$ observed for several codes. In all of them, for a given $E[L]$ and $N, w$ is selected achieving a minimum error probability. A comparison with simulated results is also presented.

The theoretical and simulated $P_{e}$ for three different expected length $(E[L]=50, E[L]=200$ and $E[L]=$ 400) are shown in Fig. 2. 


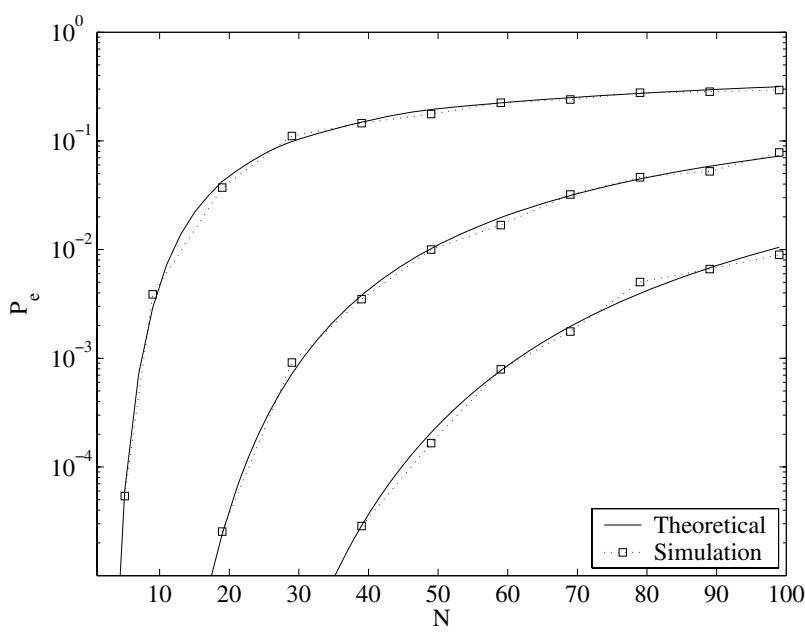

Fig. 2. Theoretical probability of error versus the number of users for a given code length. Simulation results are dotted.

The concordance between theoretical and simulated values validates the expression (15) derived in Section III. Thus, it can be used as a adequate descriptor of the system performance.

Another added value of using AOCs is the absence of limits caused by the cardinality in the number of possible users. Notice that OOCs families have important cardinality problems. The only limitations found in AOCs are caused by the Multiple-acces Interference (MAI). Its effects over the performance are well known. They can be avoided or controlled by an adequate parameter choice based on the optimization of the error probability expression (15).

\section{COMPARISON BETWEEN AOCS AND OOCS}

In this section a comparison between the performance of AOCs and OCCs with $\lambda=1$ is shown. Notice that $\lambda$ is defined for code families as $\lambda=\lambda_{c}=\lambda_{a}$. The $P_{e}$ derived in the above section for AOCs is minimized for a given $E[L]$ and $N$. It is computed choosing the $w$ where the $P_{e}$ achieves the minimum. On the other hand the $P_{e}$ for OOCs with $\lambda=1$ is optimized. For a given $E[L]$ and $N$, we choose the value of $w$ where the $P_{e}$ achieves the minimum out of the possible values. The set of all possible values is bounded by the Johnson bound [15], because the cardinality of the family should be up to $N+1$, i.e.

$$
w(w-1) \leq \frac{L-1}{N+1}
$$

The results are shown in (Fig. 3).

Fig. 3(a) and 3(b) show a comparison for a system with less than 6 interfering users. It can be observed that OOCs with $\lambda=1$ accommodates more users using short codes, achieving the same $P_{e}$. In particular they present better performances when $E[L] \leq 55$, for a $P_{e}^{\min } \leq 10^{-7}$. We can observe very different performances for longer codes. The difference in the number of users is increased with the length. We can see that OOCs behavior is only better when $E[L] \leq 80$, for a $P_{e}^{\min } \leq 10^{-10}$. This trend is confirmed in Fig. 3(c) and 3(d). AOCs present an outstanding performance in networks with several users where longer codes are required while OOCs are hardly obtainable by their low cardinality. It can be seen that, in order to get the $E[L]$ and $N$ requested, $w$ should be excessively decreased following the Johnson bound. In fact, some combinations of $E[L]$ and $N$ would be even not viable.

\section{CONCLUSION}

In this paper we have presented a new family of codes AOCs to be used in OCDMA systems. Dynamic generation of the sequence during transmission making use of a pseudo-random algorithm is the key of our proposal. This real-time generation versus pre-assignation code makes AOCs qualitative different that its preceding OOCs.

Definition, description and performance of AOCs are provided in this paper. The performance is deeply studied by the derivation of the probability of error. Expressions have been validate by the simulations results. Some of them have been shown in the paper. The method proposed presents a compromise between simplicity and performance. The most AOCs advance is to avoid the cardinality problem in optical codes design. Another added value is the perfect adaptability of the parameters to the system requirements.

\section{ACKNOWLEDGMENTS}

This work was supported in part by ESA (ESA AOE84.AO.PT.84876.05) and the Spanish Government (TEC2006-13887-C05-01/04/TCM). The authors have collaborated with other projects in CeDInt, Universidad Politecnica de Madrid.

\section{REFERENCES}

[1] F. R. K. Chung, J. A. Salehi, and V. K. Wei, "Optical orthogonal codes: Design, analysis, and applications," IEEE Trans. Inf. Theory, vol. 35, no. 3, pp. 595-604, May. 1989.

[2] J. A. Salehi, "Code division multiple-access tecniques in optical fiber networks-Part I: Fundamental principles," IEEE Trans. Commun., vol. 37, no. 8, pp. 824-833, Aug. 1989.

[3] J. A. Salehi and C. A. Brackett, "Code division multiple-access tecniques in optical fiber networks-Part II: Systems performance analysis," IEEE Trans. Commun., vol. 37, no. 8, pp. 834-842, Aug. 1989.

[4] J. A. Salehi, "Emerging optical code-division multiple-access communications systems," IEEE Netw., vol. 3, pp. 31-39, 1989.

[5] A. A. Shaar and P. A. Davies, "Prime sequences - quasi-optimal sequences for or channel code division multiplexing," Electronics Letters, vol. 19, no. 21, pp. 888-890, 1983. 


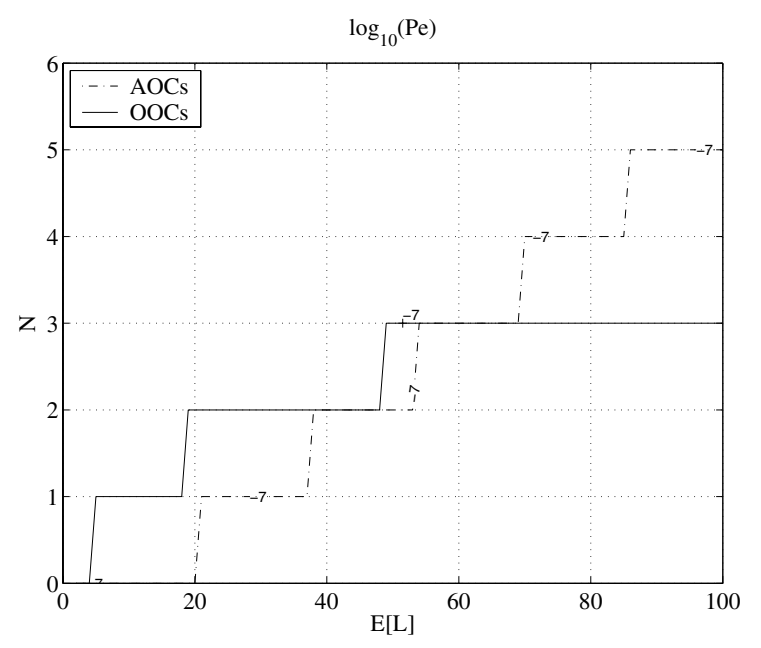

(a)

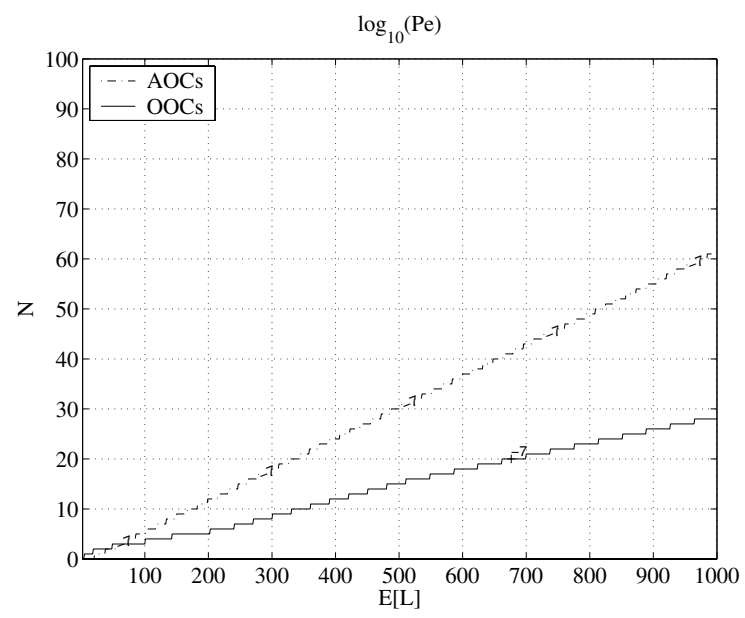

(c)

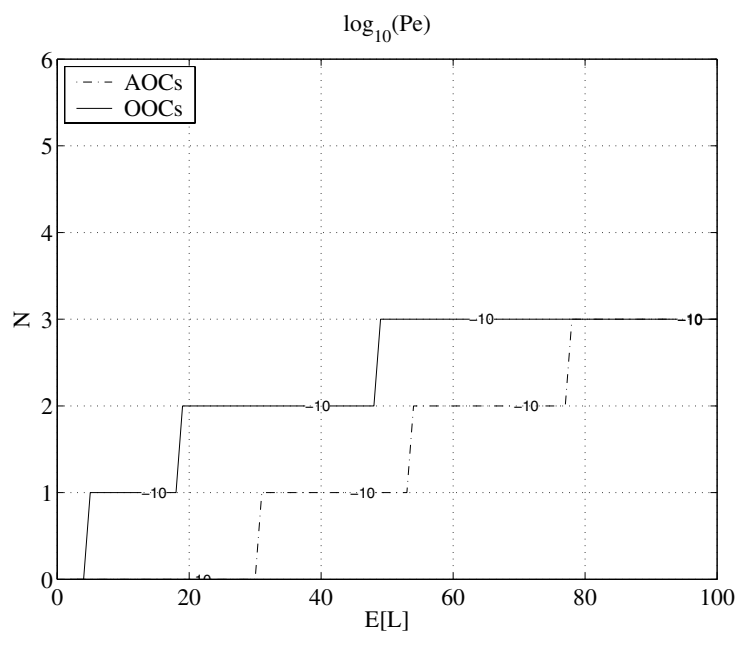

(b)

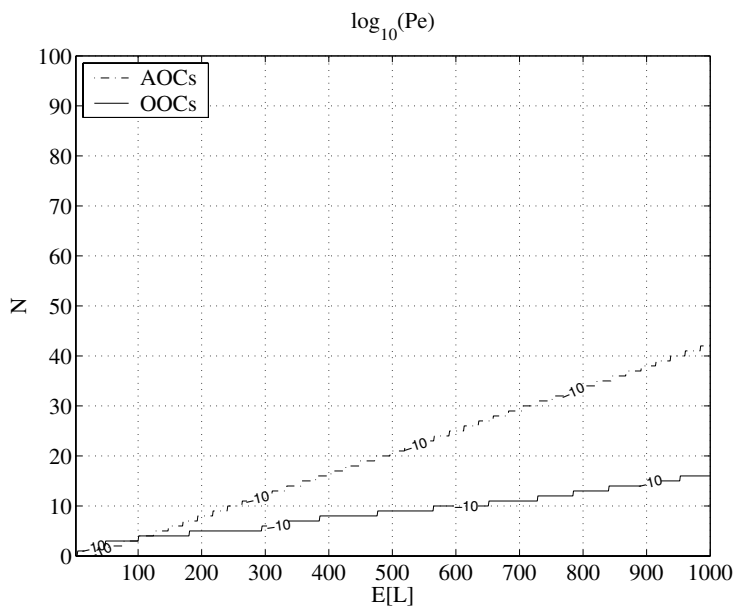

(d)

Fig. 3. Comparison on the number of simultaneous interfering users allowed for AOCs and OOCs $(\lambda=1)$ so as to obtain at less a given $P_{e}$ $\left(10^{-7}\right.$-Fig. 3(a) and 3(c)- or $10^{-10}$-Figures 3(b) and 3(d)-) as a funtion of $E[L]$ (expected value of the code length) and $N$. Figures 3(a) and 3(b) show the details of the lower values of Figures 3(c) and 3(d).

[6] P. R. Prucnal and M. A. Santoro, "Spread spectrum fiberoptic local area network using optical-processing," Journal of Lightwave Technology, vol. 4, no. 5, pp. 547-554, May. 1986.

[7] G. C. Yang and W. C. Kwong, "Performance analysis of optical CDMA with prime codes," Electronics Letters, vol. 31, no. 7, pp. 569-570, Mar. 301995.

[8] —, "On the construction of $2 \mathrm{n}$ codes for optical code-division multiple-access," IEEE Trans. Commun., vol. 43, no. 2-4, pp. 495-502, Feb.-Apr. 1995.

[9] W. C. Kwong and G. C. Yang, "Construction of 2n primesequence codes for optical code division multiple access," IEE Proceedings-Communications, vol. 142, no. 3, pp. 141-150, Jun. 1995.

[10] W. Kwong, G. C. Yang, and J. Zhang, "2n prime-sequence codes and coding architecture for optical code-division multipleaccess," IEEE Trans. Commun., vol. 44, no. 9, pp. 1152-1162, Sep. 1996.

[11] J. A. Martin-Gonzalez, E. Poves, and F. J. Lopez-Hernandez, "Random optical codes in an intra-satellite optical wireless network.” IEEE ICTON Mediterranean Winter Conference, Dec. 2007.
[12] — "Random optical codes for optical code-division multipleaccess." IASTED International Conference on Wireless and Optical Communications (WOC 2008), May. 2008.

[13] E. Poves, J. A. Martin-Gonzalez, and F. J. Lopez-Hernandez, "Use of optical orthogonal codes for intra-spacecraft communications." IEEE ICTON Mediterranean Winter Conference, Dec. 2007.

[14] J. A. Salehi, "Emerging OCDMA communication systems and data networks [invited]," Journal of Optical Networking, vol. 6, no. 9, pp. 1138-1178, Sep. 12007.

[15] S. Mashhadi and J. A. Salehi, "Code-division multiple-access techniques in optical fiber networks-Part III: Optical AND logic gate receiver structure with generalized optical orthogonal codes," IEEE Trans. Commun., vol. 54, no. 8, pp. 1457-1468, Aug. 2006. 